\title{
Results on 20 kV Spectroscopy with Monochromation and In-Column Filter
}

\author{
S. Pokrant*, A. Orchowski*, G. Benner*, M. Cheynet** and U. Kaiser*** \\ * Carl Zeiss NTS, 73447 Oberkochen, Germany \\ ** SIMAP, 38402 St. Martin d'Heres, France \\ *** Fachbereich Physik, Universität Ulm, 89069 Ulm, Germany
}

Recently, with the introduction of aberration correction [1], an increasing trend to lower acceleration energies $(<80 \mathrm{keV})$ has been initiated. The advantage of lowering the acceleration voltage lies in the reduction of beam damage (knock on damage) and the increasing scattering cross section with decreasing beam acceleration voltage, resulting in a better signal to noise ratio. Electron Energy Loss Spectroscopy (EELS) is a very interesting method in this context because of its analytical power and its reflection of electronic and optical properties in the low loss region [2]. Therefore energy filters and monochromators need to be optimized for low acceleration voltages to provide constant quality at all acceleration voltages. The aim of the Sub-Angstrom-Low-Voltage Electron Microscopy project (SALVE-project) between Ulm University, Carl Zeiss NTS and CEOS GmbH is to enable highspatial and high-energy resolution low-voltage TEM. It comprises the development of the methodology and the instrumentation for observing and analyzing beam sensitive materials (i.e. selected polymers, carbon nanotubes, molecules) or structures with very few atoms (graphene, nanoparticles) with acceleration voltages as low as $20 \mathrm{kV}$.

The advantages of working at $20 \mathrm{kV}$ in EELS are similar to the ones in TEM imaging, already clearly demonstrated by Kaiser et al. [3]: the reduction of beam damage and the increase of signal to noise. Moreover, at high acceleration voltages almost any low loss spectrum shows some kind of background in the region close to the ZLP $(\sim 1-6 \mathrm{eV})$, which is claimed to vary with sample thickness and acceleration voltage [4]. This hinders very often the interpretation of this part of the spectrum including information on the band gap and the dielectric constant [2]. Spectroscopy at $20 \mathrm{kV}$, however, promises to get rid of the background signal in the very low loss region at least partially and hence to facilitate the band gap determination and the extraction of the dielectric constant. Kaiser et al. have already shown the validity of these expectations on bulk semiconductors [2]. Since $20 \mathrm{kV}$ spectroscopy enhances the sample signal due to the increased scattering cross section, it should provide the ideal set up to study for example optical band gaps of nanoparticles.

In this contribution we will present the feasibility of monochromated spectroscopy at $20 \mathrm{kV}$ acceleration voltage with an energy resolution below $0.2 \mathrm{eV}$ (see Figure 1). The experimental set-up used is the Libra 200 FE platform including a monochromator [5] and a Cs TEM corrector. The TEM is equipped with an in-column energy filter allowing for spectroscopy down to $20 \mathrm{kV}$ acceleration voltage. The relevance of this novel type of spectroscopy will be demonstrated by applying it to semiconductor nanoparticles (see Figure 2).

\section{References :}

[1] M. Haider et al. Ultramicroscopy 75 (1998) 53.

[2] R. Egerton Electron Energy Loss Spectroscopy Plenum Press, New York, 1986

[3] U. Kaiser et al. Ultramicroscopy (2011)

[4] M. Stöger-Pollach et al. Micron 37 (2006) 396 
[5] S. Uhlenmann et al. Proc. 15 $5^{\text {th }}$ ICEM Durban S. Africa Vol 3. (2002) 327

[6] J. Park et al. Ultramicroscopy (2009) 1183, R. Erni et al. Ultramicroscopy in press

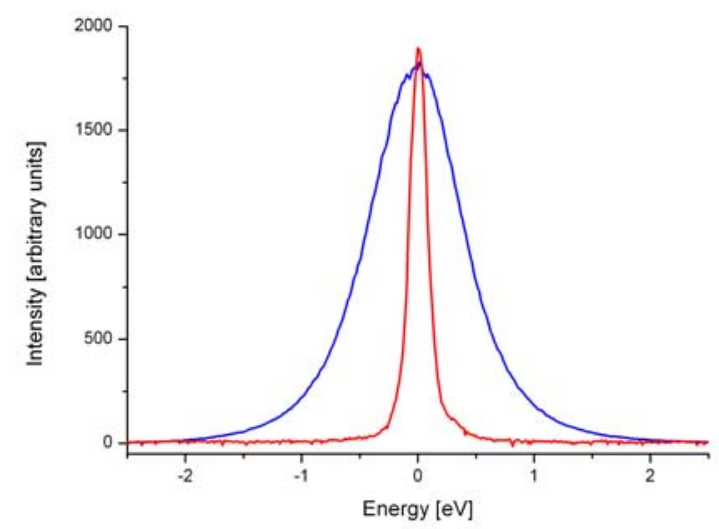

FIG. 1 a) Zero Loss Peak in the vacuum at $20 \mathrm{kV}$, in red with and in blue without monochromation. The peaks are fully symmetric. The Full Width at Half Maximum (FWHM) is $0.19 \mathrm{eV}$ and $0.93 \mathrm{eV}$ respectively.

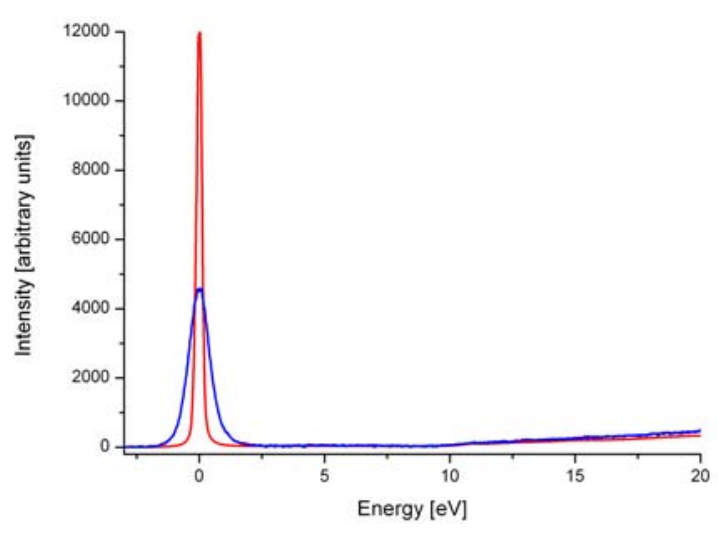

FIG.1 b) Zero Loss Peak at $20 \mathrm{kV}$ of a $15 \mathrm{~nm}$ thick $\mathrm{Si}_{3} \mathrm{~N}_{4}$ thin film, in red with and in blue without monochromation. The peaks are symmetric. The Full Width at Half Maximum (FWHM) is $0.25 \mathrm{eV}$ and $1.0 \mathrm{eV}$ respectively.

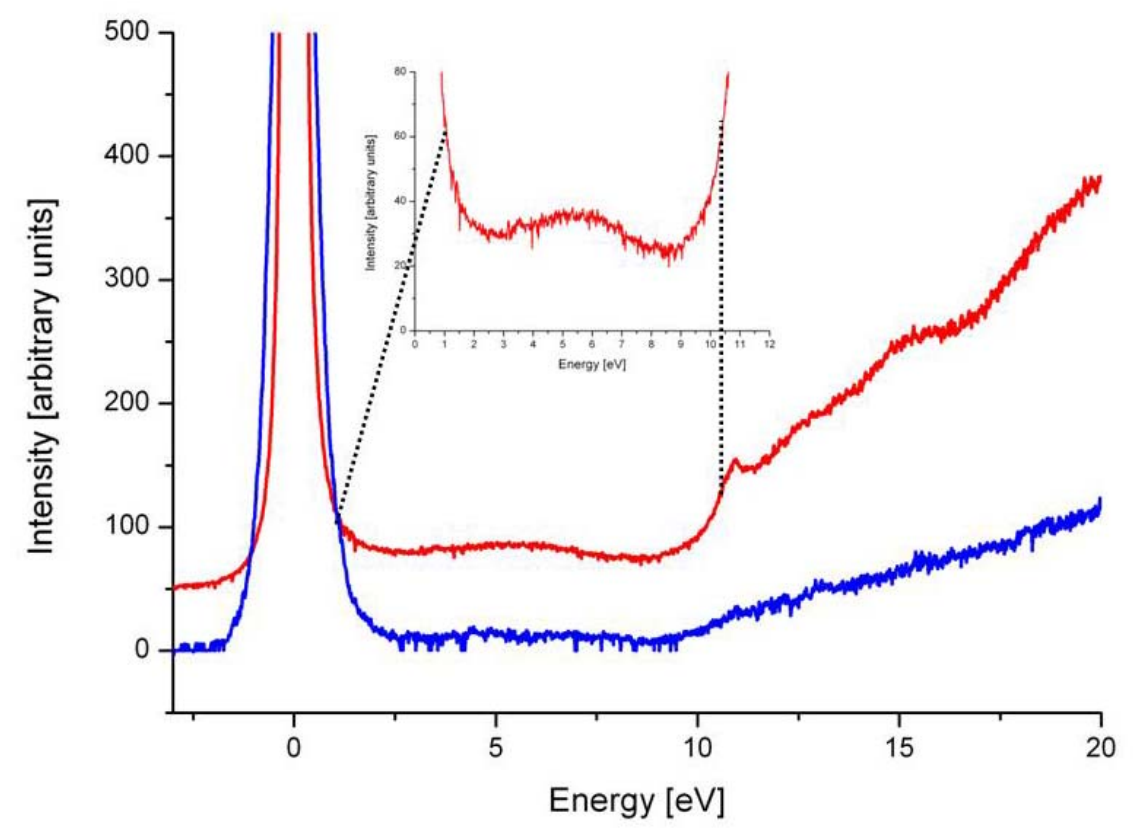

FIG. 2. Low Loss Spectrum of a $\mathrm{Si}_{3} \mathrm{~N}_{4}$ thin film with $6 \mathrm{~nm}$ PbSe nanoparticles at $20 \mathrm{kV}$, in red with and in blue without monochromation. In the inset a zoom of the low loss region between $0-10 \mathrm{eV}$ is shown. The broad peak stems very likely from the PbSe nanoparticles, since pure $\mathrm{Si}_{3} \mathrm{~N}_{4}$ does not exhibit any transitions at these energies [6]. 